\title{
The benefits of neighborhood racial diversity: neighborhood factors and its association with increased physical activity in ACS patients
}

\author{
Ellen-ge Denton, PsyD ${ }^{1}$, Philip Green, MD $^{2}$, Jonathan Newman, MD, MPH ${ }^{3}$, Ye Siqin, MD $^{2}$, \\ Karina W. Davidson, PhD $^{2}$, and Joseph Schwartz, PhD $^{2}$ \\ ${ }^{1}$ Department of Psychology, City University of New York College of Staten Island This author \\ takes responsibility for all aspects of the reliability and freedom from bias of the data presented \\ and their discussed interpretation \\ 2Department of Medicine, Columbia University Medical Center, New York, NY This author takes \\ responsibility for all aspects of the reliability and freedom from bias of the data presented and \\ their discussed interpretation \\ ${ }^{3}$ Department of Medicine, New York University Langone Medical Center This author takes \\ responsibility for all aspects of the reliability and freedom from bias of the data presented and \\ their discussed interpretation
}

\section{Keywords}

Physical activity; acute coronary syndrome; neighborhood; racial diversity

Regular physical activity reduces the risk of adverse events after an acute coronary syndrome (ACS). ${ }^{1}$ Physical activity level is influenced by neighborhood factors such as racial diversity in the general population, 2,3 but the impact of neighborhood factors on physical activity after an ACS is unknown. We therefore prospectively evaluated the relationship of post-ACS physical activity assessed by continuous activity monitors with neighborhood characteristics, including ethnic density, income, female headed households, and racial diversity, in patients enrolled in the Prescription Use, Lifestyle, and Stress Evaluation (PULSE) Study.

We included 107 patients enrolled in the PULSE study from February 1, 2009 to June 30, who were monitored with an Actical ${ }^{\circledR}$ (Philips - Respironics, Inc, Bend, Oregon) accelerometer device during the first 45 days following discharge from their ACS. For this

(C) 2014 Elsevier Ireland Ltd. All rights reserved.

Correspondence: Ellen-ge D. Denton, PsyD, Department of Psychology, City University of New York College of Staten Island, Room 4S-229 2800 Victory Boulevard Staten Island, NY 10314 Phone number: 718982 3803. Fax number: 7189824114. ellenge.denton@csi.cuny.edu..

Publisher's Disclaimer: This is a PDF file of an unedited manuscript that has been accepted for publication. As a service to our customers we are providing this early version of the manuscript. The manuscript will undergo copyediting, typesetting, and review of the resulting proof before it is published in its final citable form. Please note that during the production process errors may be discovered which could affect the content, and all legal disclaimers that apply to the journal pertain.

There are no conflicts of interest to report. 
analysis, physical activity level was operationalized as the mean maximum 6 minutes of activity during the day (M6m), which has previously been employed in studies of patients with chronic heart failure to summarize the patients' peak activity level. ${ }^{4,}{ }^{5}$ Because the trajectory of physical activity is expected to change after hospital discharge, we calculated the M6m measure at 7, 14, 21, and 28 days post-discharge.

Characteristics of neighborhood in which patients resided were determined by geocoding mailing addresses using the ArcGIS ${ }^{\mathrm{SM}}$ (Arc Geographic Information System) software to map individual patients to census tracts (Figure 1). Twenty patients were excluded, at random, as to not violate an assumption of independence when analyzing nested individuallevel and neighborhood-level data. Patients were distributed across 87 census tracts. The current analysis therefore included 87 PULSE patients. From the Census American Community Survey 2005-2009 we extracted four neighborhood characteristics corresponding to each census tract: Neighborhood racial diversity, Hispanic ethnic density, percentage female headed household, and median income. ${ }^{6,7}$ Neighborhood Racial Diversity Index is derived from the calculated variance of four racial/ethnic categories, Black, White, Asian, and Hispanic, summed together to compute the generalized measure of variance (GV), where higher values reflect a higher degree of racial/ethnic diversity. ${ }^{8}$ The study protocol conforms to the ethical guidelines of the 1975 Declaration of Helsinki as reflected in a priori approval by the Institutional Review Board of Columbia University Medical Center and all patients provided informed consent.

All neighborhood measures were investigated as continuous measures, with exception of neighborhood racial diversity, which was also categorized into quartiles with the highest quartile as reference. Using growth curve model as a base, neighborhood measures and all other covariates were individually added to the model to assess their bivariate associations with estimated physical activity (M6m) at each time point. Subsequent models assessed the independent association of neighborhood diversity with Day 7, 14, 21, and 28 physical activity after adjusting for demographic (age, gender, ethnicity, race, medicaid insurance, and education) clinical (Charlson comorbidity index, the Global Registry of Acute Coronary Events (GRACE) risk score, left ventricular ejection fracture [LVEF], body mass index, and diagnosis of diabetes mellitus) and the above-described neighborhood level predictors. Because day 7 physical activity may differ from patients Day 14, 21, and 28 physical activity, we tested for the interaction of time and time-squared with each of the significant predictors of physical activity.

The mean age of participants was 61.8 years, and the self-identified racial-ethnic composition of the patient sample is $62 \%$ White, $32 \%$ Hispanic, $19 \%$ Black, 3\% Asian, $14 \%$ Other, and $2 \%$ multiple race (Table 1). The average GV index was 0.40 , indicating that, on average, there is approximately a $40 \%$ chance that two randomly selected patients in the "average neighborhood" would belong to different racial-ethnic subgroups. After adjustment for all demographic, clinical, and neighborhood predictors, the linear association between neighborhood racial diversity and predicted peak physical activity remained significant across all time points $(\mathrm{p}<.008)$. 
Categorical analyses indicated that the predicted peak physical activity on day 28 post-ACS was on average $41.3 \%$ greater for patients living in neighborhoods in the upper quartile of neighborhood racial diversity. In the fully adjusted model, predicted peak physical activity in the most racially diverse neighborhoods was $40.6 \%, 42.5 \%, 39.6 \%$, and $32.4 \%$ greater compared to that in neighborhood in the lower three quartiles of racial diversity, at Days 7 , 14,21 , and 28 post-ACS discharge, respectively. The interaction effect of racial diversity with post-discharge day (both linear and quadratic terms) was statistically significant, indicating that the effect of neighborhood diversity on predicted peak physical activity is not constant over time.

The principal finding of this study is that neighborhood racial diversity was independently associated with higher physical activity after an ACS. On average, across all four timepoints, the upper quartile of neighborhood racial diversity had greater physical activity outcome than the bottom three quartiles of neighborhood racial diversity.

Previous studies have been inconclusive in identifying neighborhood factors associated with physical activity. Some studies suggest that lower census tract income is associated with decreased physical activity. ${ }^{2}$ On the other hand, high population density has been shown to be positively associated with physical activity. ${ }^{3}$ Also, increased residential racial segregation has been found to be associated with decreased physical activity both in the United Kingdom and in the US. ${ }^{3,10}$ Our results confirm a different association for ACS patients, increased neighborhood level of racial diversity is associated with increased peak physical activity in a largely urban area. Reasons for this association include the possibility that patients who live in racially diverse neighborhoods have differential access to facilities for physical activity, ${ }^{9}$ or that their exercise habits are positively influenced by exposure to the physical activity patterns of individuals of varying race and ethnicity.

This study was limited to a small number of participants recruited from a single center and only a subset of enrolled patients wore accelerometers. However, there were no differences across baseline characteristics between patients who enrolled in the actigraphy study versus those who did not enroll. In conclusion, these findings suggest that the environment may play an important role in post ACS activity level and merits attention when caring for patients after an ACS.

\section{Acknowledgments}

This work was supported by parent grant HL088117 and supplemental grant HL088117-02S1 from the National Heart, Lung, and Blood Institute (NHLBI) of the National Institutes of Health (NIH), Bethesda, MD., USA; and by an unrestricted research grant from the Hinduja Foundation, New York, NY.

\section{References}

1. Capewell S, Ford ES, Croft JB, Critchley JA, Greenlund KJ, Labarthe DR. Cardiovascular risk factor trends and potential for reducing coronary heart disease mortality in the united states of america. Bull World Health Organ. 2010; 88:120-130. [PubMed: 20428369]

2. Lopez RP, Hynes HP. Obesity, physical activity, and the urban environment: Public health research needs. Environ Health. 2006; 5:25. [PubMed: 16981988] 
3. Molaodi OR, Leyland AH, Ellaway A, Kearns A, Harding S. Neighbourhood food and physical activity environments in england, uk: Does ethnic density matter? Int J Behav Nutr Phys Act. 2012; 9:75. [PubMed: 22709527]

4. Howell J, Strong BM, Weisenberg J, Kakade A, Gao Q, Cuddihy P, Delisle S, Kachnowski S, Maurer MS. Maximum daily 6 minutes of activity: An index of functional capacity derived from actigraphy and its application to older adults with heart failure. J Am Geriatr Soc. 2010; 58:931936. [PubMed: 20374397]

5. Green P, Newman JD, Shaffer JA, Davidson KW, Maurer MS, Schwartz JE. Relation of patients living without a partner or spouse to being physically active after acute coronary syndromes (from the pulse accelerometry substudy). Am J Cardiol. 2013; 111:1264-1269. [PubMed: 23411104] Blau, PM.; Schwartz, JE. Crosscutting social circles. Academic Press; New York: 1984.

6. Shaw RJ. The health benefits of hispanic communities for non-hispanic mothers and infants: Another hispanic paradox. Am J Public Health. 2013; 103:1052-1057. [PubMed: 23597369]

7. Lu Y. Household migration, social support, and psychosocial health: The perspective from migrantsending areas. Soc Sci Med. 2012; 74:135-142. [PubMed: 22169626]

8. Budescu DV, Budescu M. How to measure diversity when you must. Psychol Methods. 2012; 17:215-227. [PubMed: 22309955]

9. Brownson RC, Boehmer TK, Luke DA. Declining rates of physical activity in the united states: What are the contributors? Annu Rev Public Health. 2005; 26:421-443. [PubMed: 15760296]

10. Litaker DG, Sudano JJ, Colabianchi N. Understanding the effects of racial residential segregation on health status. J Gen Intern Med. 2003; 18:180. 


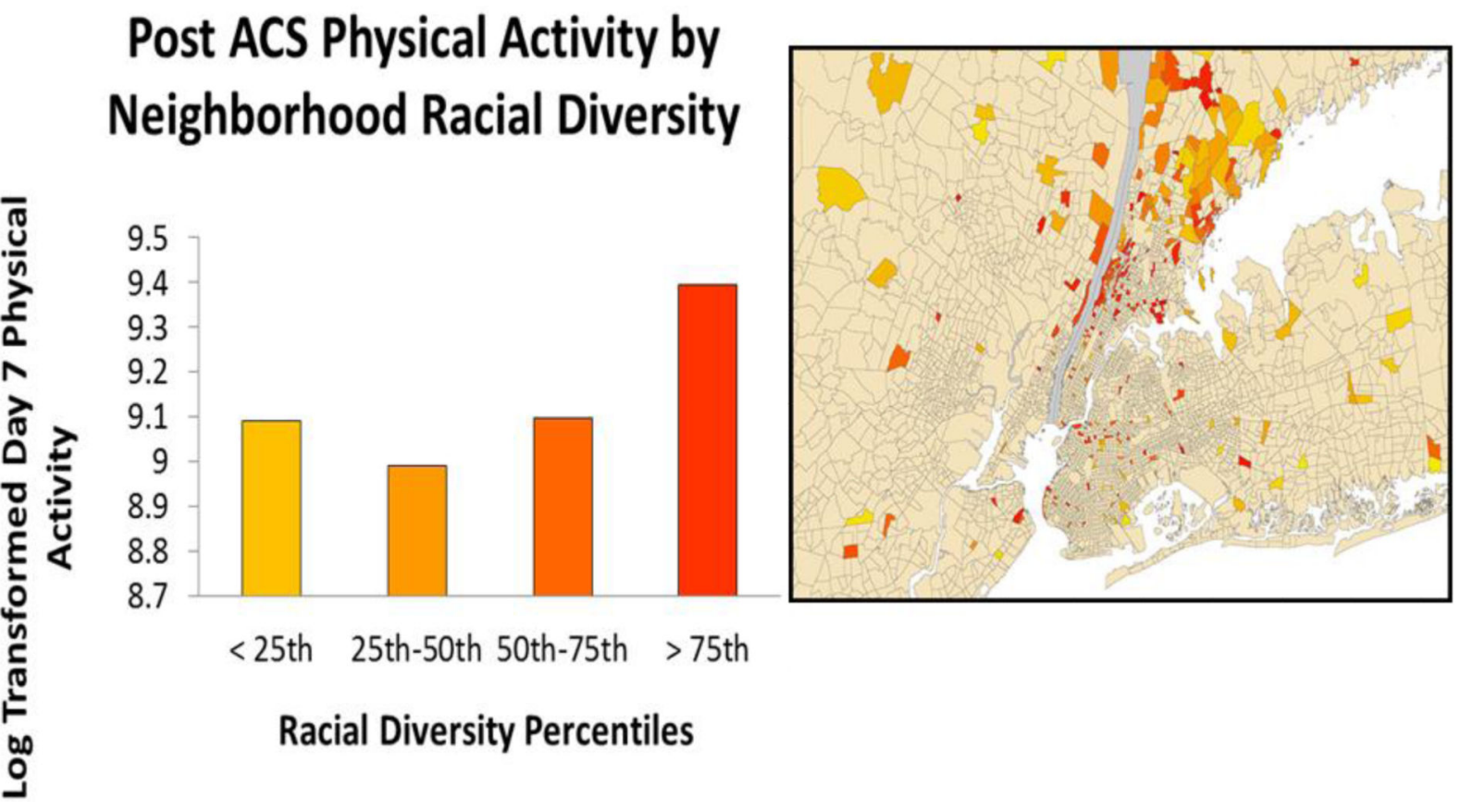

Figure 1.

Demographic mapping of neighborhood racial diversity for Post ACS patients $(\mathrm{N}=87)$. Darker colors indicate census tracts with increased racial heterogeneity. 


\section{Table 1}

Baseline Characteristics of Study Participants $(\mathrm{N}=87)$ and their Bivariate Correlations with Predicted Day 7 Peak Daytime Physical Activity

\begin{tabular}{lcc}
\hline Variable & Mean \pm SD or N (\%) & r (p value) \\
Age (years) & $61.875 \pm 12.3$ & $-.50(<.001)$ \\
Male & $59(67.8)$ & $-.26(.02)$ \\
White & $55(63.2)$ & $-.13(.22)$ \\
Education (years) & $13.3 \pm 4.0$ & $.21(.05)$ \\
Medicaid Insurance & $21(24.1)$ & $-.21(.05)$ \\
Charlson Disease severity & $1.6 \pm 1.6$ & $-.19(.09)$ \\
GRACE risk score & $86.0 \pm 29.8$ & $-.50(<.001)$ \\
LVEF (\%) & $50.7 \pm 10.7$ & $.11(.35)$ \\
BMI (Body Mass Index; kg/m $\left.{ }^{2}\right)$ & $29.3 \pm 5.5$ & $-.07(.52)$ \\
Diabetes mellitus & $30(34.5)$ & $-.11(.30)$ \\
Median Household Income & $\$ 44230 \pm 27,032$ & $-.14(.19)$ \\
Hispanic Ethnic density $(\%)$ & $27.4 \pm 28.1$ & $-.14(.19)$ \\
Female headed-household $(\%)$ & $3.3 \pm 2.5$ & $.09(.42)$ \\
Neighborhood racial diversity & $0.4+0.2$ & $.28(.01)$ \\
\hline
\end{tabular}

